\title{
Methotrexate and early postoperative complications in patients with rheumatoid arthritis undergoing elective orthopaedic surgery
}

\author{
D M Grennan, J Gray, J Loudon, S Fear
}

\begin{abstract}
Objectives-To determine whether continued methotrexate treatment increases the risk of postoperative infections or of surgical complications in patients with rheumatoid arthritis (RA) within one year of elective orthopaedic surgery.

Design-A prospective randomised study of postoperative infection or surgical complications occurring within one year of surgery in patients with RA who underwent elective orthopaedic surgery.

Subjects-388 patients with RA who were to undergo elective orthopaedic surgery. Patients who were receiving methotrexate were randomly allocated to groups who either continued methotrexate (group A) or who discontinued methotrexate from two weeks before surgery until two weeks after surgery (group B). Their complication rates were compared with complications occurring in 228 patients with RA (group C) who were not receiving methotrexate and who also underwent elective orthopaedic surgery.
\end{abstract}

Main outcome measures-Signs of postoperative infection were recorded, including rubor, discharge, systemic infection, and frequency of wound dehiscence as well as the incidence of any surgical complication requiring a secondary revision procedure that occurred within one year of surgery. The frequencies of flare up activity of RA at six weeks and six months after surgery were also recorded. A flare of rheumatoid disease was defined as an increase in joint pain in two or more joints notified by the patient as well as by an increase in articular index of at least $25 \%$ after surgery.

Results-Signs of infection or surgical complications occurred in two of 88 procedures in group $A(2 \%), 11$ of 72 procedures in group B $(15 \%)$, and 24 of 228 $(\mathbf{1 0 . 5 \% )}$ procedures in group $C$. The surgical complication or infection frequency in group $A$ was less than that in either group $B(p<0.003)$ or group $C$ $(p=0.026)$. At six weeks after surgery there were no flares in group $A$, six flares in group $B(8 \%)$, and six flares in group $C$ $(2.6 \%)$. Logistic regression analysis of the overall surgical complication rate in all the patients with RA studied showed that methotrexate, whether continued or discontinued before surgery, did not increase the early complication rate in the patients with RA who underwent elective orthopaedic surgery. Other drugspenicillamine, indometacin, cyclosporin, hydroxychloroquine, chloroquine, and prednisolone-all did significantly increase the risk of infection or surgical complication after elective orthopaedic surgery. The risk of surgery was also increased in the presence of intercurrent chronic diseases-diabetes, hypertension, bronchiectasis, psoriasis, asthma, and ischaemic heart disease.

Conclusion-Continuation of methotrexate treatment does not increase the risk of either infections or of surgical complications occurring in patients with $\mathrm{RA}$ within one year of elective orthopaedic surgery. Thus methotrexate treatment should not be stopped in patients whose disease is controlled by the drug before elective orthopaedic surgery.

(Ann Rheum Dis 2001;60:214-217)

Rheumatoid arthritis (RA) is a common and disabling condition that is now often treated by the cytotoxic drug methotrexate. ${ }^{1}$ Once the inflammation of rheumatoid disease is controlled by methotrexate, if the drug is suddenly stopped the rheumatoid disease often flares, thus making movement painful and rehabilitation and mobilisation after any surgical procedure more difficult.

A retrospective study of 53 patients with RA who underwent elective orthopaedic surgery found that in four of 19 procedures in which methotrexate treatment was continued before surgery early postoperative complications developed, as compared with no complications in 34 procedures in patients who did not receive methotrexate treatment within four weeks of surgery. ${ }^{2}$ The results of this study were widely interpreted as suggesting that methotrexate treatment might increase the risk of postoperative surgical complications in patients with RA who underwent orthopaedic surgery.

Many units, including Wrightington Hospital, initiated policies to discontinue methotrexate treatment in patients with RA receiving such treatment before elective orthopaedic surgery. However, two further retrospective and two prospective studies subsequently compared the risk of postoperative surgical complications in a further 313 procedures in patients with RA who received methotrexate treatment within four weeks of elective orthopaedic surgery and 176 patients with RA who did not receive methotrexate within four weeks of surgery. ${ }^{3-6}$ The overall results of these studies added to the results of the earlier study by 
Bridges and coworkers, ${ }^{2}$ found that 22 of 332 $(7 \%)$ procedures in subjects who continued methotrexate developed early postoperative complications, compared with 10 of 210 (5\%) procedures on patients with RA who did not receive methotrexate treatment before surgery-that is, the overall results showed a trend for an increased risk of early postoperative surgical complications in subjects who continued methotrexate before surgery.

As this is an important issue and the previous publications have reported relatively small numbers, we carried out a prospective study to compare the risk of early postoperative surgical complications in patients with RA who underwent elective orthopaedic surgery in groups who either continue or who discontinue methotrexate treatment immediately before surgery. Patients listed for revision surgery were not included in this study.

\section{Method}

SUBJECTS

We studied three groups of patients with RA who were to undergo elective orthopaedic surgery at Wrightington Hospital. Group A: patients with RA who were receiving methotrexate for at least six weeks before surgery and in whom methotrexate treatment was not discontinued. Group B: patients with RA who were receiving methotrexate matched with group A for type of surgery and in whom methotrexate treatment was stopped two weeks before surgery and restarted two weeks after surgery. Group C: patients with RA who underwent elective orthopaedic surgery during the study period and who had not received methotrexate treatment.

POSTOPERATIVE SURGICAL COMPLICATIONS We compared the incidence of early postoperative surgical complications occurring within one year of surgery in the different groups of patients. Complications were defined as wound morbidity (reddening of wound, discharge from wound), systemic infection, or wound dehiscence, loosening of implants, or any complication requiring a secondary revision procedure and occurring within one year of surgery. Samples for bacteriology were sent from all clinical infections, but a negative culture report did not alter the definition of infection for the purposes of this study. The presence or absence of infection or complication was noted at the usual postoperative reviews for each type of surgery. For the frequency of follow up for different surgical procedures see table 1 .

Table 1 Follow up for different surgical procedures

\begin{tabular}{lllccc}
\hline $\begin{array}{l}\text { Surgical } \\
\text { procedure }\end{array}$ & $1^{\text {st }}$ Follow up & $2^{\text {nd }}$ Follow up & $3^{\text {rd }}$ Follow up & $4^{\text {th }}$ Follow up & $5^{\text {th }}$ Follow up \\
\hline Shoulder & 2 weeks & 6 weeks & 3 months & 6 months & 12 months \\
Elbow & 2 weeks & 6 weeks & 3 months & 6 months & 12 months \\
Wrist & 2 weeks & 6 weeks & 3 months & 6 months & 12 months \\
MCP & 2 weeks & 6 weeks & 3 months & 6 months & 12 months \\
Other hand & 2 weeks & 6 weeks & 3 months & & \\
Hip & 3 months & 6 months & 12 months & & \\
Knee & 3 months & 6 months & 12 months & & \\
Ankle & 4 weeks & 3 months & 6 months & 12 months & \\
Other foot & 6 weeks & 3 months & 6 months & & \\
\hline
\end{tabular}

${ }^{\star} \mathrm{MCP}=$ metacarpophalangeal.
We initially set out to study 400 subjects in group A and 400 in group B over a four year period. These numbers were calculated by power calculations, using the ST plan of the National Cancer Institute Texas (1996), as the number required to have an $80 \%$ power of detecting an incidence of 40 complications in group A versus 20 in group B as significant at $\mathrm{p}=0.05$. The initial proposal stated that we would carry out a preliminary analysis at two years, but as the results of this analysis have been statistically significant we concluded the study at this two year period.

\section{PROTOCOL}

All patients with RA receiving oral methotrexate treatment who were listed for surgery at Wrightington Hospital were asked to take part in the study and all those approached agreed. We obtained the informed consent of all these patients to be randomly allocated to either group A or group B. The study protocol was approved by the Wrightington Hospital ethics committee. Patients were allocated to either group A or group B by the study coordinator, who attempted to match subjects for type of surgery and who used a block of 10 randomisation design. Surgical procedures were classified as shoulder replacements, elbow replacements, metacarpophalangeal joint replacement, wrist surgery, other hand surgery, hip surgery, knee replacements, ankle replacements, metatarsophalangeal joint surgery, or other foot surgery.

We noted and analysed the influence of sex, rheumatoid disease duration, baseline disease activity as measured by the articular index, ${ }^{7}$ Health Assessment Questionnaire score, ${ }^{10}$ the influence of other drug treatments, including penicillamine, corticosteroids, and nonsteroidal anti-inflammatory drugs, the presence of concurrent diseases such as diabetes, hypertension, osteoporosis, vasculitis, bronchiectasis, Felty's syndrome, asthma, ischaemic heart disease, and diverticulitis. Information about these disorders was obtained either from the patient's case notes or by interviewing the patient before surgery.

The articular index was measured by the same observer ${ }^{7}$ the day before surgery and at six weeks, six months, and 12 months after surgery. Presence of a rheumatoid disease flare was defined as the presence of both an increase in pain in two or more joints as noted by the patient after surgery and an increase in articular index ${ }^{7}$ by at least $25 \%$ after surgery. We examined the relation between disease flares and risk of postoperative surgical complications or infections.

\section{STATISTICS}

Differences between the groups in the overall incidence of early infection or complication were analysed by Fisher's exact test. A logistic regression analysis was used to examine the risk of other clinical or therapeutic variables on the risk of early postoperative infections or surgical complications. 
Table 2 Baseline features of subjects in groups $A-C$

\begin{tabular}{|c|c|c|c|}
\hline & Group $A$ & Group B & Group C \\
\hline Number of subjects & 88 & 72 & 228 \\
\hline Male & 16 & 16 & 40 \\
\hline Mean age (years) & 63 & 66 & 62 \\
\hline Range & $(49-73)$ & $(41-79)$ & $(38-83)$ \\
\hline Female & 72 & 56 & 188 \\
\hline Mean age (years) & 58 & 59 & 62 \\
\hline Range & $(17-84)$ & $(29-83)$ & $(20-95)$ \\
\hline \multicolumn{4}{|l|}{ RA disease duration } \\
\hline Mean (years) & & & \\
\hline Range & $(4-57)$ & $(5-50)$ & $(1-55)$ \\
\hline \multicolumn{4}{|c|}{ Baseline articular index } \\
\hline Mean & 14 & 16.5 & 15 \\
\hline Range & $0-49$ & $0-55$ & $0-61$ \\
\hline \multicolumn{4}{|l|}{$\mathrm{HAQ}^{\star}$} \\
\hline Mean & 1.9 & 1.9 & 1.8 \\
\hline Range & $0.4-3$ & $0-3$ & $0.1-3$ \\
\hline \multicolumn{4}{|l|}{ Methotrexate } \\
\hline \multicolumn{4}{|l|}{ Dose (mg) } \\
\hline Median & 10 & 7.5 & \\
\hline Range & $2.5-25$ & $2.5-20$ & \\
\hline \multicolumn{4}{|l|}{ Duration } \\
\hline Median & $3 \mathrm{yr}$ & $3 \mathrm{yr}$ & \\
\hline Range & $6 \mathrm{wk}-10 \mathrm{yr}$ & $6 \mathrm{wk}-7.5 \mathrm{yr}$ & \\
\hline
\end{tabular}

${ }^{\star} \mathrm{HAQ}=$ Health Assessment Questionnaire.

Table 3 Intercurrent diseases and drug treatments in subgroups

\begin{tabular}{|c|c|c|c|c|c|c|}
\hline \multirow[b]{2}{*}{ Intercurrent diseases } & \multicolumn{2}{|c|}{ Group $A(n=88)$} & \multicolumn{2}{|c|}{ Group $B(n=72)$} & \multicolumn{2}{|c|}{ Group $C(n=228)$} \\
\hline & No & $\%$ & No & $\%$ & No & $\%$ \\
\hline Diabetes & 5 & 6 & 5 & 7 & 6 & 3 \\
\hline Angina & 5 & 6 & 2 & 3 & 14 & 6 \\
\hline Hypertension & 3 & 3 & 6 & 8 & 12 & 5 \\
\hline Bronchiectasis & 2 & 2 & 4 & 6 & 3 & 1 \\
\hline Psoriasis & 2 & 2 & 2 & 3 & 1 & 0.4 \\
\hline Diverticulitis & 1 & 1 & 1 & 1 & 1 & 0.4 \\
\hline Asthma & 2 & 2 & 3 & 4 & 21 & 9 \\
\hline Osteoporosis & 2 & 2 & 2 & 3 & 8 & 4 \\
\hline \multicolumn{7}{|l|}{ Treatment } \\
\hline Prednisolone & 35 & 40 & 36 & 50 & 84 & 37 \\
\hline Penicillamine & 1 & 1 & 1 & 1 & 15 & 7 \\
\hline Sulfasalazine & 5 & 6 & 2 & 3 & 20 & 9 \\
\hline Gold & 2 & 2 & 0 & 0 & 14 & 6 \\
\hline Indometacin & 7 & 8 & 8 & 11 & 4 & 2 \\
\hline Hydroxychloroquine & 0 & 0 & 1 & 1 & 9 & 4 \\
\hline Chloroquine & 0 & 0 & 0 & 0 & 3 & 1 \\
\hline Cyclosporin & 0 & 0 & 1 & 1 & 1 & 0.4 \\
\hline Azathioprine & 0 & 0 & 0 & 0 & 9 & 4 \\
\hline Diclofenac & 10 & 11 & 6 & 8 & 16 & 7 \\
\hline
\end{tabular}

Table 4 Surgical procedures in subgroups

\begin{tabular}{|c|c|c|c|c|c|c|c|}
\hline \multirow[b]{2}{*}{ Surgery procedure } & \multicolumn{2}{|c|}{ Group $A(n=88)$} & \multicolumn{2}{|c|}{ Group B $(n=72)$} & \multicolumn{2}{|c|}{ Group $C(n=228)$} & \multirow[b]{2}{*}{ Total } \\
\hline & No & $\%$ & No & $\%$ & No & $\%$ & \\
\hline Shoulder & 9 & 10 & 7 & 10 & 18 & 8 & 34 \\
\hline Elbow & 1 & 1 & 2 & 3 & 25 & 11 & 28 \\
\hline Wrist & 6 & 7 & 6 & 8 & 8 & 4 & 20 \\
\hline Hip & 10 & 11 & 12 & 16 & 22 & 10 & 44 \\
\hline Knee & 18 & 20 & 14 & 19 & 45 & 20 & 77 \\
\hline Ankle & 9 & 10 & 7 & 10 & 14 & 6 & 30 \\
\hline Other foot & 7 & 8 & 5 & 7 & 23 & 10 & 35 \\
\hline Other hand & 20 & 23 & 13 & 18 & 50 & 22 & 83 \\
\hline $\mathrm{MCP}^{\star}$ & 12 & 14 & 7 & 10 & 26 & 11 & 45 \\
\hline
\end{tabular}

${ }^{\star} \mathrm{MCP}=$ metacarpophalangeal.

Table 5 Incidence of infection/complications as defined in "Methods". Results are shown as No (\%)

\begin{tabular}{llcllll}
\hline Group & Rubor & Discharge & Systemic & Dehiscence & Complication & Total \\
\hline A (88) & $0(0)$ & $1(1)$ & $0(0)$ & $1(1)$ & $0(0)$ & $2(2)$ \\
B (72) & $4(6)$ & $4(6)$ & $0(0)$ & $1(1)$ & $2(3)$ & $11(15)$ \\
C (228) & $9(4)$ & $10(4)$ & $2(1)$ & $2(1)$ & $1(0.4)$ & $24(10.5)$ \\
\hline
\end{tabular}

\section{Results}

Eighty eight procedures in group A, 72 in group B, and 228 in group $\mathrm{C}$ were studied. Tables 2 and 3 summarise the baseline features of subjects in these three groups. Table 4 summarises the surgical procedures carried out. Table 5 summarises the incidence of early infection or of complications as defined in "Methods". The incidence of infection/ complications was lower in group A than in group B (Fisher's p<0.003) and also lower than in group $C(p=0.026)$.

\section{BACTERIOLOGICAL CULTURES IN PATIENTS WITH} INFECTIONS OR COMPLICATIONS

A positive culture of mixed faecal organisms was obtained from one of the two patients with infections or complications in group A. There were no positive cultures from the 11 patients with infections or complications in group B. In group $\mathrm{C}$ in the 24 subjects with infections or complications there were cultures of staphylococci in seven, cultures of mixed faecal organisms in two, cultures of skin flora in two, and no organisms were cultured in 13.

LOGISTIC REGRESSION ANALYSIS

A detailed analysis of the factors influencing the risk of infection or surgical complications was carried out by logistic regression analysis (table 6). The presence of any of the chronic diseases analysed increased the risk of complications. Methotrexate in any dose and whether continued or discontinued before surgery did not increase the risk of surgical complications, but penicillamine, indometacin, cyclosporin, hydroxychloroquine, chloroquine, and prednisolone all did appear to increase the risk of complications.

\section{FLARES AFTER SURGERY}

Six weeks after surgery none of the patients who had continued methotrexate treatment has had a rheumatoid disease flare as compared with six $(8 \%)$ patients who stopped methotrexate and nine $(4 \%)$ of those who had not received methotrexate treatment $(p=0.04)$. There were no statistically significant differences in the incidence of flares in the different groups at six months and 12 months after surgery.

\section{Discussion}

The main aim of this study was to establish whether continuing methotrexate treatment increased the risk of early postoperative infection or complications in patients with RA who underwent elective orthopaedic surgery. Clearly, in these subjects continuing methotrexate treatment not only did not increase the duration of early postoperative infection or complication but these problems were actually fewer than in those subjects who either stopped methotrexate treatment two weeks before surgery or who had not received methotrexate treatment previously.

From the logistic regression analysis certain other drugs, such as penicillamine, indometacin, cyclosporin, antimalarial drugs, and prednisolone, appear to increase the risk of 
Table 6 Logistic regression analysis of all patients studied

\begin{tabular}{lccl}
\hline Intercurrent disease & $p$ Value & Odds ratio & $95 \%$ Confidence interval \\
\hline Diabetes & 0.005 & 4.34 & 1.57 to 11.97 \\
Hypertension & 0.03 & 3.04 & 1.14 to 8.10 \\
Osteoporosis & 0.0001 & 9.51 & 3.01 to 30.06 \\
Bronchiectasis/psoriasis/diverticulitis & $<0.0001$ & 11.12 & 3.39 to 36.57 \\
Asthma & 0.02 & 3.20 & 1.20 to 8.57 \\
Heart condition & 0.004 & 6.97 & 1.87 to 25.95 \\
& & & \\
Treatment & & & \\
Penicillamine & 0.05 & 2.64 & 1.00 to 6.94 \\
Gold & 0.56 & 1.46 & 0.41 to 5.17 \\
Azathioprine & 0.6 & 1.28 & 0.51 to 3.24 \\
Indometacin & 0.05 & 2.64 & 1.00 to 6.94 \\
Cyclosporin & $<0.0001$ & 33.78 & 6.54 to 17.44 \\
Hydroxychloroquine & 0.02 & 4.61 & 1.35 to 15.77 \\
Chloroquine & 0.003 & 30.88 & 3.13 to 305.08 \\
Sulfasalazine & 0.17 & 2.04 & 0.73 to 5.69 \\
Diclofenac & 0.3 & 1.64 & 0.64 to 4.20 \\
Prednisolone & $<0.0001$ & 21.13 & 5.00 to 89.22 \\
Leg surgery & 0.05 & 0.489 & 0.24 to 1.00 \\
Arm surgery & $<0.0001$ & 0.15 & 0.06 to 0.37 \\
Methotrexate & 0.35 & 1.12 & 0.34 to 1.40 \\
Flare up & 0.1 & 0.18 & 0.02 to 1.37 \\
Articular index & 0.22 & 0.98 & 0.94 to 1.01 \\
HAQ† & 0.15 & 1.58 & 0.84 to 2.99 \\
& &
\end{tabular}

${ }^{\star}$ Range of daily dose of prednisolone from $1 \mathrm{mg}$ to $27 \mathrm{mg}$ (mean dose $5.9 \mathrm{mg}$ ). †HAQ $=$ Health Assessment Quuestionnaire.

postoperative infections or complications. As the association between these other drugs and postoperative complications have been found as part of a coincidental analysis and not as the result of the main design of the study, the clinical significance of these associations is less certain. However, increased exposure to these drugs in groups $\mathrm{B}$ and $\mathrm{C}$ probably accounts for the apparently protective effect of methotrexate in group A (table 3).

As has been suggested in reviews ${ }^{9}$ the presence of coincidental diseases, such as diabetes and psoriasis, increases the risk of sepsis after elective arthroplasty. This effect was shown with all the chronic disorders here, including diabetes, emphasising that particular care needs to be taken to minimise the risk of sepsis in such patients. The reason for the association of increased risk of surgery with osteoporosis is unclear but might reflect an association with inherited collagen variants which could both predispose to osteoporosis and to impaired wound healing.

A limitation in the interpretation of these results is that this study was designed to look at early postoperative complications which occurred within one year of surgery and it might be argued that the decreased infection/ complication rate in subjects who continue methotrexate merely reflected an immediate anti-chemotactic effect of methotrexate. ${ }^{10} \mathrm{We}$ need to follow up our patients over the longer term to confirm whether the risk of late, deep infections is not increased in patients who continue methotrexate treatment during the operative period. However to date the one patient studied who developed a major complication at 16 days postoperatively, which ultimately resulted in an above the knee amputation after knee arthroplasty, was not receiving methotrexate treatment during the operative period. Pending analysis therefore of the longer term complication rates after elective orthopaedic surgery, in view of our results of infection or complications in the year after surgery and the fact that flares are more common when the methotrexate treatment is stopped, we conclude that methotrexate treatment should not be stopped before elective orthopaedic surgery in patients with RA whose disease is controlled by the drug before surgery.

We are grateful to the following physicians and surgeons who have allowed us to study their patients: Dr D R Swinson, Dr C Chattopadhyay, Dr P J Smith, Dr L S Teh, Mr P L R Wood, Professor M Wroblewski, Mr J Loudon, Mr M Porter, Mr A Mohammed, Mr V Raut, Mr C Faux, Mr K Hardinge, Professor J K Stanley, Mr I A Trail, Mr J H Stilwell, Dr T Hothersall.

Funding: Wishbone Trust.

Conflict of interest: None.

Contributions: Dr D M Grennan designed study and wrote the paper. Dr S Fear provided statistical advice and helped in the design and analysis of the study. E J Gray compiled the data and carried out the analysis. J Loudon helped design protocol and critically reviewed results and manuscript.

1 Kremer JF. The changing face of therapy for rheumatoid arthritis rheumatic disease. Clinics of North America arthritis rheumatic

2 Bridges S, Lopez Mendez A, Han K, Tracy F, Alarson G. Should methotrexate be discontinued before elective orthopaedic surgery in patients with rheumatoid arthritis? J Rheumatol 1991;18:984-8.

3 Kasden M, June L. Post-operative results of rheumatoid arthritis patients on methotrexate at the time of reconstructive surgery of the hand. Orthopaedics 1993;16:1233-5.

4 Sany J, Anaya J, Canovas F, Combe B, Jorgensen C, Saker S, et al. Influence of methotrexate and the frequency of postoperative infections/complications in patients with rheumatoid arthritis. J Rheumatol 1993;20:1129-32.

5 Perhala R, Wilke W, Clough J, Segal A. Local infectious complications following large joint replacements in rheumatoid arthritis patients treated with methotrexate versus those not treated with methotrexate. Arthritis Rheum 1991;34:146-52.

6 Carpenter M, West S, Jones D. Post-operative joint infections in rheumatoid arthritis patients on methotrexate infections in rheumatoid arthritis patien

7 Richie D, Boyle JA, McInnes T, Jasani M, Dalakos T, Grieveson P, et al. Clinical trials with an articular index on the assessment of joint tenderness in patients with rheumatoid arthritis. Q J Med 1958;37:393-406.

8 Fries JF, Spitz P, Kraines RG, Holman HR, Measurement of patient outcome in arthritis. Arthritis Rheum 1980;23: 1327-45.

9 Garvin K, Nebrasha O, Hanssen A. Infection after total hip arthroplasty. J Bone Joint Surg Am 1995;77:1576-87.

10 O'Callaghan J, Forrest M, Brooks P. Inhibition of neutrophil chemo-taxis in methotrexate treated rheumatoid arthritis patients. Rheumatol Int 1988; 8:41-5. 